\title{
Nutrition policies in Germany: a systematic assessment with the Food Environment Policy Index
}

\author{
Peter von Philipsborn ${ }^{1,2, *, \dagger}$, Karin Geffert ${ }^{1,2, \dagger}$, Carmen Klinger ${ }^{1,2}$, Antje Hebestreit ${ }^{3}$, \\ Jan Stratil ${ }^{1,2}$ and Eva Annette Rehfuess ${ }^{1,2}$ for the PEN Consortium \\ 'Chair of Public Health and Health Services Research, Institute for Medical Information Processing, Biometry, \\ and Epidemiology (IBE), LMU Munich, Munich 80539, Germany: ${ }^{2}$ Pettenkofer School of Public Health, Munich, \\ Germany: ${ }^{3}$ Leibniz Institute for Prevention Research and Epidemiology - BIPS, Bremen, Germany
}

Submitted 13 August 2021: Final revision received 2 December 2021: Accepted 4 December 2021: First published online 9 December 2021

\begin{abstract}
Objective: To systematically assess Germany's nutrition policies, to benchmark them against international best practices and to identify priority policy actions to improve population-level nutrition in Germany.

Design: We applied the Food Environment Policy Index (Food-EPI), a methodological framework developed by the International Network for Food and Obesity/ non-communicable Diseases Research, Monitoring and Action Support (INFORMAS) network. Qualitative content analysis of laws, directives and other documents formed the basis of a multistaged, structured consultation process. Setting: Germany.

Participants: The expert consultation process included fifty-five experts from academia, public administration and civil society.

Results: Germany lags behind international best practices in several key policy areas. For eighteen policy indicators, the degree of implementation compared with international best practices was rated as very low, for twenty-one as low, for eight as intermediate and for none as high. In particular, indicators on food taxation, regulation of food marketing as well as retail and food service sector policies were rated as very low to low. Identified priority actions included the binding implementation of nutrition standards for schools and kindergartens, a reform of the value added tax on foods and beverages, a sugar-sweetened beverage tax and stricter regulation of food marketing directed at children.

Conclusions: The results show that Germany makes insufficient use of the potential of evidence-informed health-promoting nutrition policies. Adopting international best practices in key policy areas could help to reduce the burden of nutritionrelated chronic disease and related inequalities in nutrition and health in Germany. Implementation of relevant policies requires political leadership, a broad societal dialogue and evidence-informed advocacy by civil society, including the scientific community.
\end{abstract}

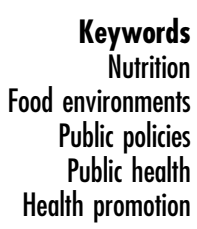

Nutrition Puvironments Public health Health promotion
Unhealthy dietary patterns are among the most important preventable risk factors for disease and premature death worldwide $^{(1)}$. In particular, unhealthy diets can increase the risk for obesity, type 2 diabetes mellitus, CVD and certain cancers $^{(1,2)}$. Approximately $8 \%$ of the global burden of disease is attributed to dietary risks ${ }^{(1,3)}$. Over the past decades, global dietary patterns have undergone fundamental shifts, with an increase in the per-capita

†eter von Philipsborn and Karin Geffert have contributed equally to this work and are shared first authors. consumption of ultra-processed foods and beverages with a high content of sugar, refined grains, fat and salt ${ }^{(4-6)}$. These shifts have contributed to a rising burden of dietrelated chronic disease ${ }^{(7)}$. In particular, the global prevalence of obesity has doubled from $6 \%$ to $13 \%$ among adults between 1985 and $2016^{(8)}$ and risen sevenfold from $1 \%$ to $7 \%$ among children between 1975 and 2016(9). Similarly, the global prevalence of type 2 diabetes mellitus has roughly doubled from 5\% to 9\% between 1980 and $2014^{(10)}$. 
Individual dietary intake is strongly influenced by the food environment, defined as the collective physical, economic, policy and sociocultural surroundings, opportunities and conditions that influence people's food and beverage choices and nutritional status ${ }^{(11)}$. The food environment is shaped, among others, by public policies in areas such as food composition, labelling, taxation, marketing and public procurement. The evidence on the effectiveness of public policies supporting healthy diets has grown considerably over the past years, but implementation remains highly uneven across countries ${ }^{(12-14)}$.

Unhealthy diets and diet-related diseases are also a key public health issue in Germany. In Germany's National Nutrition Survey II (NVS II), conducted in a nationally representative sample from 2005 to 2007, significant deviations from the recommended consumption were found for most food groups ${ }^{(15)}$. Follow-up studies conducted from 2008 to 2015 as part of the longitudinal National Nutrition Monitoring did not detect relevant improvements over time ${ }^{(16)}$. Consumption levels of vegetables, meat and sausages and dairy products remained largely unchanged, while fruit consumption decreased and confectionery consumption increased $^{(16)}$. The prevalence of obesity in Germany is $23 \%$ among adults and $6 \%$ among children and is therefore above the European average ${ }^{(17)}$. While the prevalence of obesity has remained stable among children since $2003^{(18)}$, it continues to increase among adults, in particular among low socio-economic status groups ${ }^{(19)}$. Obesity and its underlying dietary risk factors are also associated with high costs for the health care system. For instance, direct health-related costs of excessive consumption of sugar, fat and salt in Germany were estimated to account for nearly 17 billion euros, or $7 \%$ of all direct health care costs in $2008^{(20)}$.

Against this background, the German government has implemented or announced a number of measures to support healthier diets on the population level ${ }^{(21,22)}$. These include a National Reformulation and Innovation Strategy for Sugar, Salt and Fat in Processed Foods; the introduction of the Nutri-Score nutrition labelling system on a voluntary basis; the founding of a new national nutrition education and information centre (Bundeszentrum für Ernährung, or BZfE) and measures to improve the quality of food served in schools and kindergartens through information and training ${ }^{(21-23)}$.

To the authors' knowledge, the current nutrition-related policy landscape in Germany has, however, not yet been assessed in a comprehensive, systematic and international comparative manner. Internationally, a number of approaches for the comprehensive assessment of the degree of implementation of nutrition policies have been proposed $^{(11,24,25)}$. One of the most widely used approaches is the Food Environment Policy Index (Food-EPI), which has been developed by INFORMAS (International Network for Food and Obesity/non-communicable Diseases Research, Monitoring and Action Support) based on the review and evaluation of existing approaches and the involvement of stakeholders from policy and practice ${ }^{(11,26,27)}$. INFORMAS is a global network of researchers and public interest organisations that aims to monitor, benchmark and support public and private sector actions to support healthy food environments and reduce obesity and non-communicable diseases and their related inequalities. It has received funding from a variety of public sources, including the International Development Research Centre, which is funded by the Canadian government ${ }^{(28)}$.

To date, the Food-EPI has been applied in around forty countries worldwide ${ }^{(29)}$. In the present paper, we use the Food-EPI framework to systematically assess the current nutrition policy landscape in Germany, benchmark it against international best practices for health-promoting nutrition policies and identify priority actions for reform. This work was conducted within the Policy Evaluation Network (www.jpi-pen.eu) as a project of the Joint Programming Initiative 'A Healthy Diet for a Healthy Life ${ }^{(30)}$. Funding was provided by Germany's Federal Ministry of Education and Research (grant number 01EA1818I).

\section{Methods}

The Food-EPI framework and its development have been described in greater detail elsewhere ${ }^{(11)}$. In short, the FoodEPI is a policy analysis framework based on a qualitative content analysis of relevant documents, as well as a structured, multi-stage expert consultation process. It includes thirteen domains, seven of which describe substantive policy areas (such as labelling and taxation), while six describe overarching structures and supportive functions (such as monitoring and surveillance, governance and funding). For each domain, two to five indicators are defined, resulting in a total of forty-seven indicators (see Table 1 for an overview, and the supplementary appendix for a more detailed description). The focus is on policies relevant for the promotion of healthy diets that minimise the risk of chronic, nutrition-related conditions such as obesity and type 2 diabetes mellitus. Aspects of food safety (i.e. prevention of microbiological, chemical or physical contaminations and food-borne infections) are excluded, as are the promotion of breast-feeding and the regulation of dietary supplements. Aspects of environmental sustainability and animal welfare are also not covered. Policies to address social inequalities in nutrition, as well as approaches to reduce food insecurity among socially disadvantaged population groups are covered (e.g. through indicators 4.4, 8.5, 10.6, 13.1 and 13.2). However, policies to address widespread hunger and undernutrition, which persist in many low- and middle-income countries, are not systematically covered with specific indicators. Work is under way in the INFORMAS network to further improve 
Table 1 Benchmarking results for Germany. Results show the experts' assessment of the degree of implementation in Germany relative to international best practices. A detailed description of the current implementation in Germany and international best practices was published separately ${ }^{(32)}$

Description (domain or indicator)

Level of implementation

1. Food composition

Food composition standards for processed foods

Food composition standards for out-of-home meals

2. Food labelling

Ingredient lists and nutrient declarations

Regulatory systems for health and nutrition claims

Front-of-pack nutrition labelling

Nutrition labelling in the food service sector (e.g. menu board labelling)

3. Regulation of food marketing

Rules for broadcast media (radio and TV)

Rules for the internet including social media

Rules for other media (e.g. print media, leaflets and direct mailings)

Rules for settings in which children congregate (e.g. schools)

Rules for product design and packaging

4. Food prices

Taxes on healthy foods

Taxes on less healthy foods

Food subsidies

Promotion of healthy diets through food-related income-support (e.g. food banks)

5. Food provision in public institutions and on worksites

Nutrition standards for educational settings (including schools and kindergartens)

Nutrition standards for other public institutions

Support and training for the implementation of nutrition standards in public institutions

Support and training for the implementation of nutrition standards in private companies

6. Food retail

Zoning laws on unhealthy food outlets (e.g. fast food restaurants)

Zoning laws on healthy food outlets (e.g. green grocers, farmers' markets)

Support for healthy in-store food environments in food retail

Support for healthy food environments in the food service sector

7. International trade and investment

Health impact assessments on trade and investment agreements

Consideration of health and nutrition aspects in trade and investment agreements

8. Political leadership and official dietary guidelines

Strong visible political support for healthy diets

Intake targets and reference values for key nutrients and food groups

Food-based dietary guidelines

Action plan for healthy food environments

Targets for reducing social inequalities in nutritional status and intake

9. Governance (including management of conflicts of interest and use of evidence)

Measures to restrict commercial influence on policy development

Structures and procedures for using evidence in food policy development

Transparency and access to governmental information

10. Monitoring und surveillance

Monitoring food environments

Monitoring nutritional status and intake

Monitoring overweight and obesity

Monitoring other NCD risk factors (e.g. diabetes mellitus)

Evaluation of programmes and policies

Monitoring of societal inequalities in nutrition and health

11. Funding

Public funding for the promotion of healthy diets

Public funding for nutrition research

Statutory agency with secure funding tasked with promoting healthy diets

12. Platforms for interaction between government, academia, civil society and the food industry

Co-ordination between government levels and portfolios

Exchange between government and the commercial food sector

Exchange between government, civil society and academia

13. Inter-sectoral approaches (health in all policies)

Systems-based approach to supporting healthy diets

Assessment and consideration of public health effects of policies across government departments 
the framework's applicability to low- and middle-income countries.

The country-level implementation of the Food-EPI involves the following steps, described in greater detail in the subsequent paragraphs:

1. Adaptation of the international Food-EPI framework to the national context

2. Identification of relevant policies and compilation of descriptive information on these in an evidence report

3. Establishment of an expert panel, including researchers and representatives of relevant government bodies and civil society organisations

4. Validation of the evidence report by the expert panel

5. Assessment of the degree of implementation for each indicator in comparison with international best practice examples by the expert panel (benchmarking)

6. Identification and prioritisation of policy recommendations by the expert panel

In conducting and reporting our research, we followed the Consolidated Criteria for Reporting Qualitative Studies (COREQ) $)^{(31)}$. The COREQ Checklist is provided in Supplemental Table S5 in the supplementary appendix.

\section{Adaptation of the Food-Environment Policy Index to the national context}

We identified six indicators in the international Food-EPI framework that we found to be largely overlapping in the German context, and which we therefore merged into three pairs, as shown in Supplemental Table S4 in the supplementary appendix.

\section{Identification of relevant policies and compilation of descriptive information}

To identify relevant policies, we searched for policyrelated, publicly available documents (such as laws, directives, official reports and other government documents, position statements, press releases and minutes of parliamentary debates). Any document providing substantive information on any of the Food-EPI indicators was included. We searched the following websites manually, with Google Advanced Search and through site-specific search functions, using search terms related to nutrition and obesity prevention:

- The websites of federal ministries, regulatory agencies and administrative bodies in the areas of nutrition and health (i.e. the Federal Ministry of Health, the Federal Ministry of Nutrition and Agriculture, the Federal Centre for Nutrition, the Robert Koch Institute, the Max Rubner Institute and the Federal Centre for Risk Assessment)

- The website of the Bundestag, Germany's parliament, and its archive of parliamentary debates, motions and inquiries
- The websites of non-governmental organisations, including professional organizations, trade associations and pressure groups

- German and European legal databases (N-Lex and EU-LEX)

- International policy databases maintained by the WHO (i.e. the WHO NCD Document Repository, the Global Database on the Implementation of Nutrition Action and the e-Library of Evidence for Nutrition Actions) and scientific associations (the NOURISHING database by the World Cancer Research Fund International, and the Global Obesity Observatory by World Obesity)

We used the data analysis software MAXQDA (VERBI Software $\mathrm{GmbH}$ ) to conduct a deductive qualitative content analysis of the included documents, using the domains and indicators of the Food-EPI as coding framework. Data were coded independently by two authors (PvP and KG). Results were then summarised narratively, and, where appropriate, illustrated with quotes. The information gathered through this analysis was complemented by consulting existing literature (e.g. textbooks on German and European food law and academic publications on nutrition policies in Germany) and through targeted Google searches. All information was fully referenced to ensure transparency. The process was guided by the aim to provide a comprehensive, yet succinct description of the current legal framework as well as existing government policies, programmes, projects and initiatives for each of the forty-seven Food-EPI indicators.

In the process of data collection and analysis, we focused on the federal (i.e. national) level in Germany. However, we also included information on EU regulations and programmes applicable to Germany - complemented, where available, by information on how these are implemented nationally. In policy areas where the main regulatory authority lies with the subnational (state or local) level, we aimed to provide an overview of relevant policies on these levels, too, e.g. by stating how many of Germany's sixteen states have policies on a specific issue, or by providing illustrative examples.

The information gathered was compiled in an evidence report, including references to all sources used ${ }^{(32)}$.

\section{Recruitment of an expert panel}

For the subsequent steps, we recruited an expert panel, including academics and experts from professional and scientific associations as well as civil society and governmental organisations. To avoid conflicts of interest, no industry representatives were involved. We used purposive sampling to identify relevant experts, based on the authors' knowledge, research and contacts, as well as recommendations by included experts. We sought to recruit experts from all fields of nutrition, with a focus on individuals with expertise in nutrition policies and public 
health nutrition. Besides, we sought to achieve a balance between researchers and experts working in policy and practice. Participants were contacted by email and provided with an information sheet (see supplementary appendix).

\section{Validation of the evidence report}

To ensure that the information compiled in the evidence report is complete, accurate and up to date, we sent a draft to the members of the expert panel, asking them to provide feedback in the form most convenient to them (e.g. using the comment and track-changes mode of their word processor, or by email or telephone). We then revised the draft evidence report based on their feedback.

\section{Benchmarking}

The next step involved the benchmarking, i.e. the assessment, for each indicator, of the degree of implementation in Germany as compared with international best practices. The selection of international best practice examples was based on a consensual collection produced by INFORMAS and updated within the Policy Evaluation Network. For application in Germany, a selection of relevant examples was taken from this collection by the authors and complemented with examples from the NOURISHING database of the World Cancer Research Fund International ${ }^{(33)}$.

For the benchmarking, the participating experts were provided with a table allowing, for each of the forty-seven indicators, a direct comparison of existing policies in Germany (as identified and described in the evidence report) with the international best practice examples. The experts could rate the degree of implementation in Germany on a four-point Likert scale as very low, low, medium or high. The rating could be completed on paper or online, with the online version allowing for anonymous data submission. For a summary assessment of the degree of implementation, we calculated the median of the ratings provided by the participating experts for each indicator.

\section{Identification and prioritisation of policy recommendations}

We searched existing catalogues of recommendations by the WHO and national professional and scientific associations and compiled a draft list of relevant policy recommendations for Germany. This list was sent to the members of the expert panel for feedback and suggestions on additional policy recommendations. Subsequently, a revised list of recommendations was discussed with the expert panel during a 2-h online workshop. We recorded and transcribed the workshop, using the transcript for further revisions of the draft list of recommendations. Analogous to the structure of the Food-EPI framework, recommendations were divided into two areas: policy actions, such as a tax on sugar-sweetened beverages, and infrastructure support actions, such as improved monitoring and surveillance.

Based on the final list of recommendations, the expert panel prioritised policy actions and infrastructure support actions according to two criteria - (i) their contribution to improving nutrition at the population level and (ii) their achievability, defined as the likelihood of policy adoption and the feasibility of legal and administrative implementation in Germany. Policy actions were additionally prioritised according to a third criterion, (iii) their contribution to improving nutrition in socially disadvantaged population groups. For the prioritisation, each recommendation could be given up to five points per criterion, with the total number of points to be distributed per criterion limited to twice the number of recommendations. For example, there were ten recommendations on infrastructure support actions, implying that participating experts could distribute up to twenty points across the ten recommendations for the criterion of impact and twenty points for the criterion of achievability. For data analysis, we calculated the arithmetic mean of the points awarded by the participating experts to each recommendation for each criterion. To arrive at an overall ranking of recommendations, we calculated a summary score for each recommendation by summing up the scores for the individual criteria.

\section{Results}

\section{Expert response rate and composition of the expert panel}

We contacted seventy-two experts, fifty-five of whom agreed to participate. A list of participating experts including their affiliations is provided in Supplemental Table S1 of the supplementary appendix. About 53\% ( $n$ 29) of participating experts were based in academia, $27 \%(n 15)$ in professional and scientific associations and civil society organisations and $20 \%$ ( $n$ 11) in government agencies. One participant reported receiving lecture and reviewer fees from food companies as a relevant conflict of interest; for the others, no relevant conflicts of interest were identified. Of the fifty-five participating experts, $91 \%$ ( $n$ 50) provided feedback on the evidence report, $67 \%$ ( $n$ 37) participated in the benchmarking of policies and $53 \%$ ( $n$ 29) in the prioritisation of policies.

\section{Existing policies and their benchmarking against international best practices}

A detailed description of existing policies in Germany based on a total of 341 documents reviewed and analysed, as well as the international best practice examples used in the benchmarking exercise, were published separately in German ${ }^{(32)}$. Table 1 presents results of the benchmarking across the thirteen Food-EPI domains, i.e. food composition, food labelling, regulation of food marketing, 
food prices, food provision in public institutions and on worksites, food retail, international trade and investment, political leadership and official dietary guidelines, governance, monitoring and surveillance, funding, platforms for interaction between government, academia, civil society and the food industry and inter-sectoral approaches. For eighteen indicators, the degree of implementation in Germany relative to international best practices was rated as very low, for twenty-one as low, for eight as intermediate and for none as high. The lowest ratings were observed for regulation of food advertisement and marketing, food pricing, promotion of healthy food choices in retail settings and intersectoral approaches. The highest ratings were noted for political leadership and official dietary guidelines and monitoring and surveillance.

\section{Identification and prioritisation of policy recommendations}

We identified a total of eighteen recommendations on policy actions and ten recommendations on infrastructure support actions (see Tables 2 and 3). A detailed description of these is provided in Tables S2 and S3 in the supplementary appendix, including a full list of references. The following policy actions were ranked highest with regard to their impact on improving population-level nutrition (see Table 2): a health-promoting reform of the value added tax (VAT) (i.e. lower tax rates on healthier foods and beverages and higher rates on less healthy ones); mandatory nutrition standards for schools and kindergartens; regulation of marketing of unhealthy foods and beverages towards children and a tax on sugar-sweetened beverages. Regarding achievability, the following policy measures were ranked highest: mandatory nutrition standards for schools and kindergartens; an action plan on the promotion of drinking water and nutrition education in schools and kindergartens (see Table 2). Figure 1 illustrates that some of the policy actions with the greatest potential for improving population-level nutrition were assessed to be relatively more achievable (e.g. mandatory nutrition standards for schools and kindergartens), whereas the implementation of others is likely to be more challenging (e.g. a health-promoting VAT and mandatory reformulation of processed foods). Most policies with a potential to improving population-level nutrition were also considered to be likely to reduce inequalities in nutrition and health.

For infrastructure support actions, the following recommendations were considered to have the largest potential positive impact on population-level nutrition (see Table 3): evaluation of interventions and policies; monitoring of nutritional status and dietary behaviour, as well as knowledge transfer between policy, practice and research. The highest achievability ratings were given to the evaluation of interventions and policies, as well as local networks and initiatives.

Table 2 Priority recommendations on policy actions to improve population-level nutrition in Germany, based on expert judgement. Higher scores/darker colours indicate a higher priority

\begin{tabular}{|c|c|c|c|c|}
\hline & Priority recommendations on policy actions & $\begin{array}{l}\text { Contribution to improving } \\
\text { population-level nutrition }\end{array}$ & Achievability & $\begin{array}{l}\text { Reduction of } \\
\text { inequalities in nutrition } \\
\text { and health }\end{array}$ \\
\hline M1 & Mandatory nutrition standards for schools and kindergartens & $2 \cdot 9$ & $2 \cdot 9$ & 3.5 \\
\hline M2 & $\begin{array}{l}\text { Health promoting VAT (reduced value added tax rate on healthy } \\
\text { foods and increased value added tax rate on less healthy } \\
\text { foods) }\end{array}$ & $3 \cdot 1$ & $1 \cdot 9$ & $3 \cdot 0$ \\
\hline M3 & Introduction of a SSB tax & $2 \cdot 7$ & $2 \cdot 3$ & $2 \cdot 7$ \\
\hline M4 & $\begin{array}{l}\text { Regulation of marketing of unhealthy foods and beverages } \\
\text { towards children }\end{array}$ & $2 \cdot 9$ & $2 \cdot 0$ & $2 \cdot 6$ \\
\hline M5 & $\begin{array}{l}\text { Mandatory nutrition standards for public institutions (other than } \\
\text { schools and kindergartens) }\end{array}$ & $2 \cdot 5$ & $2 \cdot 4$ & $2 \cdot 2$ \\
\hline M6 & Action plan on the promotion of drinking water & $2 \cdot 2$ & $2 \cdot 7$ & $2 \cdot 0$ \\
\hline M7 & School fruit and vegetable programme & 1.6 & 2.4 & $2 \cdot 8$ \\
\hline M8 & Nutrition education in kindergartens and schools & $2 \cdot 1$ & 2.4 & $2 \cdot 2$ \\
\hline M9 & Mandatory implementation of the Nutri-Score & $2 \cdot 2$ & $2 \cdot 3$ & $1 \cdot 8$ \\
\hline M10 & $\begin{array}{l}\text { Reformulation of processed food (including mandatory } \\
\text { regulations) }\end{array}$ & $2 \cdot 3$ & $1 \cdot 6$ & $2 \cdot 3$ \\
\hline M11 & Improvement of nutrition standards for private companies & 1.8 & $2 \cdot 0$ & 1.7 \\
\hline M12 & Municipal food policy measures & 1.4 & $2 \cdot 0$ & 1.3 \\
\hline M13 & Regulation of food advertising in general & 1.8 & $1 \cdot 1$ & 1.7 \\
\hline M14 & Regulations for retail outlets & 1.6 & 1.5 & 1.4 \\
\hline M15 & Reduction of portion and packaging sizes & 1.3 & 1.3 & 1.6 \\
\hline M16 & Effective regulation of health claims & $1 \cdot 1$ & 1.7 & $0 \cdot 8$ \\
\hline M17 & $\begin{array}{l}\text { Mandatory nutrition labelling on menus of quick service } \\
\text { restaurants }\end{array}$ & $1 \cdot 1$ & $1 \cdot 3$ & 0.7 \\
\hline M18 & Quality standards for children's meals in restaurants & 0.8 & $1 \cdot 1$ & 0.6 \\
\hline
\end{tabular}

VAT, value added tax; SSB, sugar-sweetened beverages. 


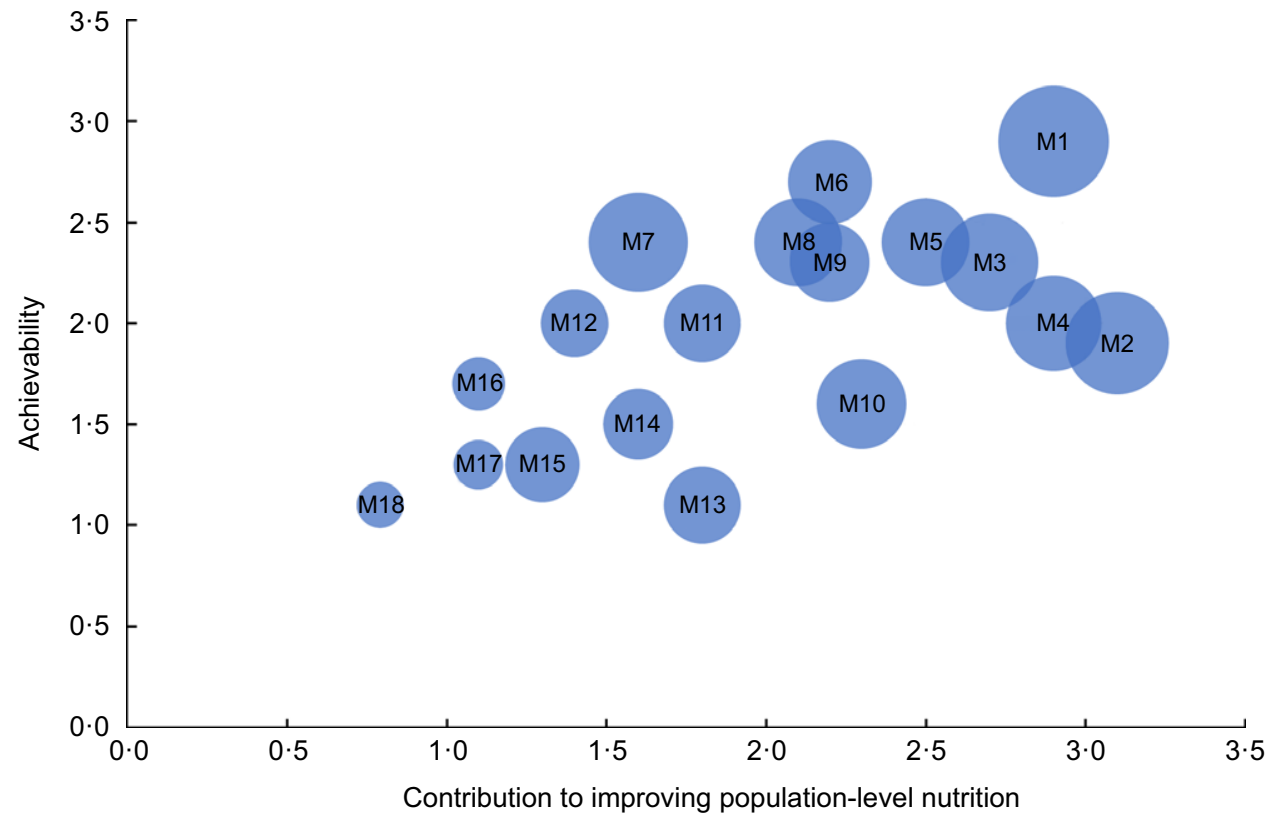

Fig. 1 (colour online) Results of the prioritisation of policy actions. The codes M1-M18 are explained in Table 2. The size of the dots represents the scores on criterion 3 (contribution to reducing social inequalities in nutrition and health)

Table 3 Priority recommendations for infrastructure support actions to improve population-level nutrition in Germany, based on expert judgement. Higher scores indicate a higher priority

\begin{tabular}{|c|c|c|c|}
\hline & Priority recommendations for infrastructure support actions & $\begin{array}{l}\text { Contribution to improving } \\
\text { population-level nutrition }\end{array}$ & Achievability \\
\hline S1 & Evaluation of interventions and policies & $2 \cdot 7$ & $2 \cdot 3$ \\
\hline S2 & Monitoring of nutritional status and dietary behaviour & $2 \cdot 1$ & $2 \cdot 0$ \\
\hline S3 & Knowledge transfer between policy, practice and research & $2 \cdot 1$ & 1.9 \\
\hline S4 & Nutrition education in the curricula of relevant professions & 1.9 & $2 \cdot 0$ \\
\hline S5 & Monitoring of food environments & $2 \cdot 0$ & 1.9 \\
\hline S6 & Local networks and initiatives & 1.6 & $2 \cdot 2$ \\
\hline S7 & Action plan for healthy nutrition & 1.6 & $2 \cdot 0$ \\
\hline S8 & Nutrition policy capacity building & 1.9 & 1.6 \\
\hline S9 & Improving the availability of data & 1.6 & 1.7 \\
\hline S10 & $\begin{array}{l}\text { Capacities for the further development of nutrition } \\
\text { recommendations }\end{array}$ & 1.6 & $1 \cdot 7$ \\
\hline
\end{tabular}

\section{Discussion}

The policy analysis using the Food-EPI framework reveals strengths and weaknesses of Germany's nutrition policy landscape. The results of the international benchmarking indicate that in some policy areas Germany has strong, institutionalised structures for promoting healthy nutrition. This applies, amongst others, to the derivation of official food-based dietary guidelines and nutrition targets; the existence of publicly funded bodies with secured funding tasked with promoting healthy diets and public commitment by political leaders. However, in other areas, Germany lags significantly behind current international best practices. This is particularly the case with regard to the regulation of food advertising and marketing; the consideration of health aspects in the taxation and subsidisation of foods; the promotion of healthy food supplies in retail; as well as cross-sectoral health-in-allpolicies approaches.

\section{Policy implications}

The identification and prioritisation of policy recommendations indicate how policymakers could address the shortcomings of Germany's nutrition policy framework revealed by the benchmarking. The results of our prioritisation exercise are in line with existing recommendations from expert groups and professional and scientific associations, including the Scientific Advisory Board at Germany's Federal Ministry of Food and Agriculture and the German Alliance for Non-communicable Diseases $^{(34,35)}$. The mandatory, nationwide, and publicly financed implementation of the existing nutrition standards for schools and kindergartens was rated the highest across 
the three prioritisation criteria population impact, achievability and contribution to reducing inequalities. This requires government subsidies for running costs, as well as investments in canteens and dining halls. For this purpose, a federal investment programme has been proposed, which could be implemented as part of the German and EU COVID-19 recovery programme ${ }^{(34)}$. The policy measures ranked the second and third highest were a health-promoting value-added tax on foods and beverages and a tax on sugar-sweetened beverages. Although health-related food and beverages taxes - sugar-sweetened beverages taxes in particular - have gained momentum across the globe ${ }^{(36)}$, so far the German government has been reluctant to consider this approach. However, it may gain traction in light of the fiscal implications of the COVID-19 pandemic, and a number of policymakers and political parties in Germany have come forward in support of the use of economic instruments in nutrition policy ${ }^{(37)}$. Similarly, the policy recommendation ranked the fourth highest - a stricter regulation of food marketing directed towards children - has recently received considerable attention from policymakers in Germany ${ }^{(38)}$.

Many of the priority actions identified in our study could be complemented by action on the EU level, or would even need changes of EU regulation to be fully implemented. For example, the health-promoting VAT reform - the second highest ranked policy recommendation in our study would ideally involve a reduction of the VAT on healthy foods such as fruit and vegetables from the current rate in Germany of $7 \%$ to the lowest possible rate of $0 \%$. However, EU regulation currently requires a minimum VAT rate of $5 \%$, limiting the use of the tax system for health promotion. Indeed, in the application of the Food-EPI on the EU level, conducted as part of a separate project, the recommendation to allow Member States to lower the VAT to $0 \%$ for healthy foods was ranked third-highest ${ }^{(39)}$. In general, recent analyses have found that the EU does not make full use of its potential to support healthy diets ${ }^{(39,40)}$.

This study also has important policy implications for Germany's sixteen states (the Länder) as well as its approximately 11000 municipalities. In Germany's federal system, responsibility for most policy fields is shared across administrative levels. This gives states and municipalities leeway in adopting a number of priority policy measures identified in this study, independent of whether such measures are being advanced or not by the Federal Government. For example, some states, including Saarland and the city-state of Berlin, have adopted mandatory nutrition standards for some parts of their school systems, while others have adopted such standards for canteens in government offices ${ }^{(32)}$. Many municipalities run childcare services and hospitals, which allows them to implement nutrition standards in these settings.

The results show the potential for policy learning from international best practices. Based on the assessment of our expert panel, Germany does not reach a high level of implementation relative to international best practices for any of the forty-seven indicators and very low to low levels for a majority of indicators. This shows the large potential for strengthening Germany's policy framework for improving food environments and population-level nutrition by adopting current international best practices.

In sum, this study may inform government policy across all political levels. Besides, it can inform advocacy work by civil society organisations and policy advice provided by scientific and medical organisations. Research has shown that advocacy and advisory work by civil society, including scientific and medical organisations, can be crucial for the adoption and implementation of evidence-informed nutrition policies ${ }^{(41)}$.

\section{Strengths, challenges and limitations}

The strengths of the present work include the use of a comprehensive, rigorous, internationally harmonised methodological framework; high standards in the conduct and reporting of the research, in line with the Consolidated Criteria for Reporting Qualitative Studies ${ }^{(31)}$; an extensive analysis of the current policy landscape in Germany and its clear and detailed presentation in the form of an evidence report; the comparison of the degree of implementation in Germany with current international best practices, i.e. with a benchmark that has already been achieved in practice in other countries and the inclusion of the expertise of a wide circle of experts from academia, public administration and civil society who were consulted in a multi-stage and multimodal process, as well as the high response rate.

A challenge in applying the Food-EPI framework to Germany was its federal structure with responsibilities distributed across various levels (municipalities, sixteen states, the federal government and the EU). In measuring and assessing the degree of implementation, we also considered policies, programmes and initiatives on the level of municipalities and states but less comprehensively than on the federal level. For policies and programmes on the EU level, we assessed their extent and the way they are implemented in Germany.

The results of the Food-EPI are derived from the reasoned judgement of experts based on a systematic and comprehensive compilation and analysis of relevant data. It therefore inevitably involves a certain degree of subjectivity. Alternative assessment methods, as well as a different selection of participating experts, may have produced divergent results, at least in detail. The benchmarking was carried out in July 2020 and the prioritisation from November 2020 to January 2021. Some of the policy areas covered by our analysis are developing dynamically, and evaluations can therefore quickly become out of date. For example, implementation of indicator 9.1 (Measures to restrict commercial influence on policy development) was assessed to be very low. Indeed, Germany's Federal 
Ministry of Nutrition and Agriculture has in the past been criticised by civil society groups for having close ties with food industry actors and for being unduly influenced by their interests ${ }^{(42)}$. However, a number of policies relevant to this indicator have been announced or adopted since the assessment took place in July 2020. This includes a public, mandatory register of professional lobbyists seeking to influence the work of the Bundestag, Germany's parliament ${ }^{(43)}$, as well as a code of conduct for lobbyists seeking to influence the work of Germany's federal government ${ }^{(44)}$.

The Food-EPI focuses on the analysis of the influence of nutrition on human health. However, social, environmental and animal welfare aspects play an important role in food policy, too ${ }^{(34)}$. Due to limited capacities and the complexity of these issues, it was not possible to consider these aspects extensively within the scope of this project, but it should be considered for future projects.

\section{Conclusions}

The present analysis shows that the nutrition policy landscape in Germany features a number of strengths, but also substantial room for improvement. Existing policies and structures were rated as particularly low in the following areas: the regulation of food advertising, food taxation, the promotion of a healthy food supply in retail and cross-sectoral approaches. Priority actions to address these shortcomings include a mandatory implementation of nutrition standards for schools and kindergartens; a health-promoting reform of the value added tax and a tax on sugar-sweetened beverages; as well as stricter regulation of food marketing targeting children. Efforts by a broad range of actors, including policy makers, civil society and academia, are required to support action on these priority measures for promoting healthy nutrition at the population level.

An earlier version of this work was published as a preprint on medRxiv (doi: https://doi.org/10.1101/ 2021.10.11.21264774), and as a German-language project report and policy brief ${ }^{(45,46)}$.

\section{Acknowledgements}

Acknowledgements: We would like to acknowledge the substantial and generous contributions made by the fiftyfive external experts, listed in the supplementary appendix, who comprised the expert panel that informed the present work. We would also like to thank the Policy Evaluation Network (www.jpi-pen.eu) as well as INFORMAS for providing protocols used in this research. Financial support: This work was funded by a grant from Germany's Federal Ministry for Education and Research as part of Joint Programming Initiative 'A Healthy Diet for a Healthy Life' (Grant number 01EA1818I). The funder had no role in the design, analysis or writing of this article. Conflicts of interest: There are no conflicts of interest. Authorship: P.vP. conceived the idea for the paper and led the research. P.vP. and K.G. developed the methodology, collected and analysed the data, interpreted the results and wrote the first draft. C.K. contributed to data collection, analysis, interpretation of results and manuscript preparation. A.H., J.S. and E.A.R. contributed to the development of the methodology, interpretation of results and manuscript preparation. Ethics of buman subject participation: Ethics approval was granted by the ethics committee of the LMU Munich.

\section{Supplementary material}

For supplementary material $/ \mathrm{s}$ referred to in this article, please visit https://doi.org/10.1017/S1368980021004742

\section{References}

1. Afshin A, Sur PJ, Fay KA et al. (2019) Health effects of dietary risks in 195 countries, 1990-2017: a systematic analysis for the Global Burden of Disease Study 2017. Lancet 393, 1958-1972.

2. NCDRC (2019) NCD Risk Factor Collaboration. http:// ncdrisc.org/ (accessed October 2019).

3. IHME (2019) Institute for Health Metrics and Evaluation. GBD Results Tool. http://ghdx.healthdata.org/gbd-resultstool (accessed October 2019).

4. Popkin BM, Corvalan C \& Grummer-Strawn LM (2020) Dynamics of the double burden of malnutrition and the changing nutrition reality. Lancet 395, 65-74.

5. Baker P, Machado P, Santos T et al. (2020) Ultra-processed foods and the nutrition transition: global, regional and national trends, food systems transformations and political economy drivers. Obes Rev 21, e13126.

6. Pingali P (2007) Westernization of Asian diets and the transformation of food systems: implications for research and policy. Food Policy 32, 281-298.

7. Vandevijvere S, Chow CC, Hall KD et al. (2015) Increased food energy supply as a major driver of the obesity epidemic: a global analysis. Bull World Health Organ 93, 446-456.

8. World Health Organization (2017) Prevalence of Obesity among Adults, BMI $\geq 30$, Age-Standardized. Estimates by WHO Region. Global Health Observatory Data Repository. https://apps.who.int/gho/data/view.main.REGION2480A? lang=en (accessed June 2021).

9. Abarca-Gómez L, Abdeen ZA, Hamid ZA et al. (2017) Worldwide trends in body-mass index, underweight, overweight, and obesity from 1975 to 2016: a pooled analysis of 2416 population-based measurement studies in 128.9 million children, adolescents, and adults. Lancet 390, 2627-2642.

10. NCD Risk Factor Collaborators (2016) Worldwide trends in diabetes since 1980: a pooled analysis of 751 populationbased studies with 44 million participants. Lancet 387, 1513-1530.

11. Swinburn B, Vandevijvere S, Kraak V et al. (2013) Monitoring and benchmarking government policies and actions to improve the healthiness of food environments: a proposed Government Healthy Food Environment Policy Index. Obes Rev 14, 24-37. 
12. Mozaffarian D, Angell SY, Lang T et al. (2018) Role of government policy in nutrition - barriers to and opportunities for healthier eating. BMJ 361, k2426.

13. Vandevijvere S, Barquera S, Caceres G et al. (2019) An 11-country study to benchmark the implementation of recommended nutrition policies by national governments using the Healthy Food Environment Policy Index, 2015-2018. Obes Rev 20, 57-66.

14. Breda J, Castro LSN, Whiting S et al. (2020) Towards better nutrition in Europe: evaluating progress and defining future directions. Food Policy 96, 101887.

15. Hilbig A, Heuer T, Krems C et al. (2009) What does Germany eat? Evaluation of the Second National Diet Study (NSV II). Ernäbrungs-Umschau: Forschung \& Praxis 56, 16-23.

16. Max Rubner Institut (2019) Longitudinal study NEMONIT. www.mri.bund.de/de/institute/ernaehrungsverhalten/ forschungsprojekte/nemonit/ (accessed November 2019).

17. OECD (2019) OECD Health Policy Studies: The Heavy Burden of Obesity - The Economics of Prevention. www. oecd.org/health/the-heavy-burden-of-obesity-67450d67-en. htm (accessed November 2021).

18. Schienkiewitz A, Damerow S, Schaffrath Rosario A et al. (2019) Body mass index among children and adolescents: prevalences and distribution considering underweight and extreme obesity. Results of KiGGS Wave 2 and trends. Bundesgesundheitsblatt - Gesundheitsforschung Gesundheitsschutz 62, 1225-1234.

19. Hoebel J, Kuntz B, Kroll LE et al. (2019) Socioeconomic inequalities in the rise of adult obesity: a time-trend analysis of National Examination Data from Germany, 1990-2011. Obes Facts 12, 344-356.

20. Meier T, Senftleben K, Deumelandt P et al. (2015) Healthcare costs associated with an adequate intake of sugars, salt and saturated fat in Germany: a health econometrical analysis. PLoS One 10, e0135990.

21. von Philipsborn P, Drees S, Geffert K et al. (2018) Disease prevention and health promotion in Germany: a qualitative analysis of the Federal Goverment's Coalition Agreement. Das Gesundheitswesen 80, e54-e61.

22. von Philipsborn P, Drees S, Geffert K et al. (2019) Prevention of obesity and diabetes mellitus: a mid-term evaluation after two years of government. Adipositas 13, 160.

23. von Philipsborn P, Effertz T, Laxy M et al. (2018) Prevention of obesity and diabetes mellitus as a political challenge (article in German). Adipositas 12, 113-119.

24. Hawkes C, Jewell J \& Allen K (2013) A food policy package for healthy diets and the prevention of obesity and dietrelated non-communicable diseases: the NOURISHING framework. Obes Rev 14, 159-168.

25. Vandevijvere S \& Swinburn B (2014) Towards global benchmarking of food environments and policies to reduce obesity and diet-related non-communicable diseases: design and methods for nation-wide surveys. BMJ Open 4, e005339.

26. INFORMAS (2018) INFORMAS Protocols. http://www. informas.org/protocols/ (accessed January 2018).

27. INFORMAS (2017) INFORMAS Protocol: Public Sector Module. Healthy Food Environment Policy Index (Food-EPI). https://figshare.com/articles/INFORMAS_Protocol_Public_ Sector_Module_-_Healthy_Food_Environment_Policy_Index_ Food-EPI_5673439 (accessed March 2020).

28. INFORMAS (2021) What is INFORMAS? https://www. informas.org/about-informas/ (accessed July 2021).

29. INFORMAS (2018) INFORMAS Countries. www.fmhs. auckland.ac.nz/en/soph/global-health/projects/informas/ regions.html (accessed August 2018).

30. Lakerveld J, Woods C, Hebestreit A et al. (2020) Advancing the evidence base for public policies impacting on dietary behaviour, physical activity and sedentary behaviour in Europe: the Policy Evaluation Network promoting a multidisciplinary approach. Food Policy 96, 101873.
31. Tong A, Sainsbury P \& Craig J (2007) Consolidated criteria for reporting qualitative research (COREQ): a 32-item checklist for interviews and focus groups. Int J Qual Health Care 19, 349-357.

32. von Philipsborn P, Geffert K, Klinger C et al. (2021) Food Environment Policy Index (Food-EPI) Evidenzbericht für Deutschland. https://www.jpi-pen.eu/images/reports/FoodEPI_Germany_Evidence_Report.pdf (accessed May 2021).

33. World Cancer Research Fund International (2019) NOURISHING Framework. https://www.wcrf.org/int/ policy/nourishing/our-policy-framework-promote-healthydiets-reduce-obesity (accessed January 2019).

34. Wissenschaftlicher Beirat für Agrarpolitik Ernährung und gesundheitlichen Verbraucherschutz (WBAE) beim BMEL (2020) Promoting more sustainable food consumption. Developing an integrated food policy and creating fair food environments. https://www.bmel.de/SharedDocs/Down loads/DE/_Ministerium/Beiraete/agrarpolitik/wbae-gutachtennachhaltige-ernaehrung.html (accessed September 2020).

35. Schaller K, Effertz T, Gerlach S et al. (2016) Prevention of non-comunicable diseases - a whole-of-society challenge (DANK). www.dank-allianz.de/files/content/dokumente/ DANK-Grundsatzpapier_ES.pdf (accessed November 2021).

36. Popkin BM \& Ng SW (2021) Sugar-sweetened beverage taxes: lessons to date and the future of taxation. PLoS Med 18, e1003412.

37. von Philipsborn P, Heise TL, Lhachimi SK et al. (2017) Obesity prevention: time for a tax on sugar-sweetened beverages. Deutsches Ärzteblatt 114, A160-164.

38. von Philipsborn P (2021) Marketing of children's foods: problem statement and policy options. https://www.vzbv. de/sites/default/files/downloads/2021/02/16/vzbv_philipsb orn_bericht_kindermarketing_2021-02.pdf (accessed February 2021)

39. Djojosoeparto S, Kamphuis C, Vandevijvere S et al. (2021) The Healthy Food Environment Policy Index (Food-EPI): European Union. An Assess EU-Level Policies Influencing Food Environments and Priority Actions to Create Healthy Food Environments EU. https://www.jpi-pen.eu/images/reports/ Food-EPI_EU_FINAL_20210305.pdf (accessed June 2021).

40. De Schutter O, Jacobs N \& Clément C (2020) A 'Common Food Policy' for Europe: how governance reforms can spark a shift to healthy diets and sustainable food systems. Food Policy 96, 101849.

41. von Philipsborn P, Garlichs D, Wildner M et al. (2020) Political implementation of population-level prevention: challenges and success factors. Gesundheitswesen $\mathbf{8 2}(05)$, 386-388.

42. Foodwatch (2021) Secret lobby meetings of Julia Klöckner: foodwatch pursues legal action. https://www.foodwatch. org/de/aktuelle-nachrichten/2021/geheime-lobbytreffen-vonjulia-kloeckner-foodwatch-klagt/ (accessed November 2021).

43. Deutscher Bundestag (2021) Parlamentarians vote for lobby register for the Bundestag. https://www.bundestag.de/ dokumente/textarchiv/2021/kw12-de-lobbyregister-798182 (accessed November 2021).

44. Bundesministerium des Inneren (2021) Federal cabinet adopts code of conduct for lobbyists. https://www.bmi. bund.de/SharedDocs/kurzmeldungen/DE/2021/06/verhalt enskodex-lobbyregistergesetz.html (accessed November 2021).

45. von Philipsborn P, Geffert K, Klinger C et al. (2021) The Food Environment Policy Index (Food-EPI) Report for Germany. www.jpi-pen.eu/images/reports/Food-EPI_Erg ebnisbericht_V11.pdf (accessed November 2021).

46. von Philipsborn P, Geffert K, Klinger C et al. (2021) Policy Brief: Policies for Healthy Diets. Status Quo and Recommendations for Germany. www.jpi-pen.eu/images/ reports/Food-EPI_PolicyBrief_V8.pdf (accessed November 2021). 